\title{
应用资源选择函数研究朱噮的巢址选择
}

\author{
李欣海 ${ }^{1}$ 马志军 ${ }^{2}$ 李典谟 $^{1}$ 丁长青 $^{1}$ 翟天庆 $^{3} \quad$ 路宝忠 ${ }^{3}$ \\ 1(中国科学院动物研究所, 北京 100080) \\ 2 (复旦大学生物多样性研究所, 上海 200433) \\ 3 (陕西省朱噮保护观察站，陕西洋县 723300)
}

摘要: 本文介绍了资源选择函数的原理与方法, 并分析了朱噮 (Nipponia nippon) 的巢址选择。我们将上述结果与主 成分分析的结果进行了比较, 发现两种方法都显示水田面积、营巢树高度、海拔高度和人类干扰程度对朱堽巢址选 择有较大影响, 而巢向、巢上郁闭度、坡向和坡度对朱鹆影响较小。然而,两种方法也有明显的差异:资源选择函数 显示营巢地的坡位非常重要,而主成分分析显示植被密度比较重要。分析表明资源选择函数更好地反映了多种生 境因素对朱鹆巢址选择的影响。本文探讨了应用资源选择函数所必须注意的对照样方选择和参数的独立性问题, 并提出了对朱噮保护工作的建议。

关键词：朱囬，资源选择函数，主成分分析，巢址选择

中图分类号:Q958 文献标识码：A 文章编号 : 1005-0094(2001) 04-0352-07

\section{Using Resource Selection Functions to study nest site selection of Crested Ibis}

LI Xin-Hai ${ }^{1}$, MA Zhi-Jun ${ }^{2}$, LI Dian-Mo ${ }^{1}$, DING Chang-Qing ${ }^{1}$, ZHAI Tian-Qing ${ }^{3}$, LU Bao-Zhong ${ }^{3}$ 1 Institute of Zoology, Chinese Academy of Sciences, Beijing 100080

2 Institute of Biodiversity, Fudan University, Shanghai 200433

3 The Crested Ibis Conservation Station, Yang County, Shaanxi 723300

Abstract : We introduced the theory and methodology of Resource Selection Functions ( RSFs) , and used a RSF to analyze nest site selection of Crested Ibis (Nipponia nippon). We compared the results of the RSF with those of principle component analysis (PCA), and found that both methods indicated that habitat factors such as area of rice paddy, height of nest tree , elevation and human disturbance played important roles in nest site selection. Nest orientation, coverage above the nest, aspect and slope of the hill where the nest tree was located were also not important. However, there were significant differences between the two methods. The RSF showed that the position of the nest tree on the slope greatly affected nest site selection, and PCA indicated that the tree density was more important. After detailed comparison, we concluded that RSFs are better than the commonly used PCA in studying habitat selection. We also discuss issues about how to select control plots and the independence of parameters while using RSFs. Finally we offer some suggestions for the conservation of Crested Ibis.

Key words : Crested Ibis , Resource Selection Functions (RSFs), principle component analysis (PCA), nest site selection

野生动物的生境选择是一个复杂的过程, 涉及 到食物、安全、理化条件等多种因素, 以往通常用各 种描述性的方法进行研究 (高中信等, 1992)。主成 分分析作为一种多元统计方法也经常被使用 (Wen- ny et al. ,1993; Liang \& Thomson, 1994; Pogue \& Schnell, 1994）,但它侧重用少数几个主成分尽可能 多地保留原始变量的信息, 而不着重于野生动物的 生境偏好。Neu et al. (1974) 和 Byers et al. (1984) 
分别比较了栖息地利用程度和可获得程度，反映了 野生动物对每个生境因子的偏好,并在生境选择研 究中得到了较广泛的应用 (马志军等,2000) ,但他 们的方法没有权衡不同因子间的权重。Alldredge \& Ratti $(1986,1992)$ 曾详细地比较了研究野生动物 资源选择的不同方法,着重分析了它们不同的假设 和统计检验方法。Manly et al. (1993) 提出了用资 源选择函数 (Resource Selection Functions，简称 RSFs) 研究栖息地利用的方法, 系统分析了野生动物对 每个生境因子的偏好及其综合影响，在近年来的生 态学研究中得到越来越多的应用 (Boyce \& McDonald，1999)。最近人们又发展了一些量化的模型 来改进资源选择函数（Mladenoff \& Sickley，1998）， 使它可以很好地配合地理信息系统中的生境数据， 人们可以比较方便地应用资源选择函数计算物种的 生境偏好和在生境斑块中的种群大小, 大大促进了 野生动物栖息地利用的研究 (Boyce \& McDonald, 1999)。

朱噮曾高度濒危, 濒临灭绝( 李欣海等,1996)。 近年来在人们的大力保护下, 朱槶种群大小逐渐回 升,由 1981 年的 7 只发展到 2001 年的 155 只。但 是，因朱揋栖息地面积并未成比例地显著扩大，所以
朱㽣密度明显增加。因此, 它依旧对污染、干旱等局 部灾害非常敏感,没有摆脱濒危的境地。为了婏救 朱㟪, 扩大它的栖息地面积, 并在适宜的地方进行再 引入工程, 我们研究了朱揋的巢址选择规律, 分析了 制约朱㽣生境利用的多种因素。

\section{1 研究方法}

\section{1 数据的采集和处理}

本研究调查的 35 个巢址均位于陕西省洋县境 内 $\left(107^{\circ} 23^{\prime} 15.12^{\prime \prime} \sim 107^{\circ} 28^{\prime} 59.82^{\prime \prime} \mathrm{E}, 33^{\circ} 18^{\prime} 39.9^{\prime \prime}\right.$ $\left.\sim 33^{\circ} 24^{\prime} 47.64^{\prime \prime} \mathrm{N}\right)$, 分布于秦岭南坡海拔 $680 \sim$ $1200 \mathrm{~m}$ 的山区。该地区人口密度较小, 交通闭塞, 环境幽静。森林覆盖率在 $60 \%$ 以上, 优势种为马尾 松 (Pinus massoniana) 和栓皮栋 (Quercus variablils) 等。由于温度较低, 作物一年只能一熟。河边有一 定面积的冬水田, 环境基本未受污染。朱鲴的巢一 般建在山坡中部树林中较高大的乔木上, 有时也建 在山坡的顶部或底部。朱㛱也在村边的独立树上营 巢。巢一般建在距地面十余米的柇树或马尾松的侧 枝上, 有时借用上年的旧巢。不同巢附近的地形、营 巢树高度、巢向和巢上郁闭度也有不同程度的差异 (表 1)。

表 1 影响朱噮果址选择的生境因子间的相关性

Table 1 The correlations between the habitat factors that affect nest site selection of Crested Ibis

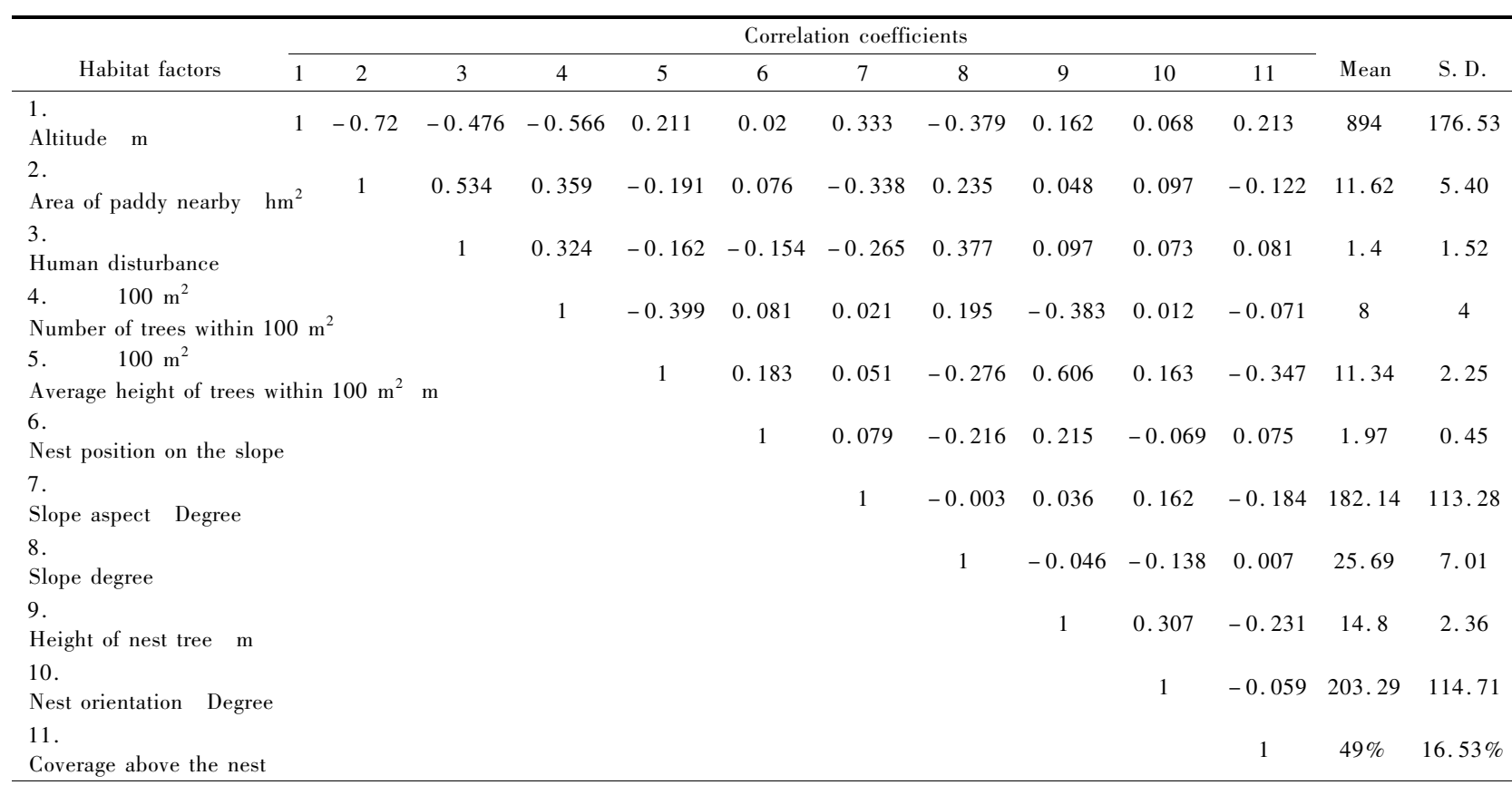


我们应用样方调查获取朱噮巢址的生境信息。 我们测量了 35 个朱愄巢址的生境参数，包括海拔高 度、营巢树高度、巢向、巢上郁闭度、坡位、坡向、坡 度、附近水田面积、营巢树周围 $100 \mathrm{~m}^{2}$ 树数量和高 度以及人类干扰程度。其中海拔高度指营巢树下地 面的海拔高度;巢向指巢相对于树干的方向, 取值为 以树干为轴, 自正北方向顺时针旋转至巢所转过的 度数坡位指营巢树所在山坡的位置, 坡顶、坡中和 坡底取值分别为 1 、 2 和 3 ; 坡向指营巢树所在山坡 所正对的方向，取值为自正北方向顺时针旋转至山 坡所正对的方向所转过的度数;附近水田面积指附 近 $1 \mathrm{~km}^{2}$ 范围内的水田面积，因为朱愄的领域面积 为 $1 \mathrm{~km}^{2}$ 左右 ${ }^{1}$,所以该参数表示朱䀦领域范围内的 水田面积;周围 $100 \mathrm{~m}^{2}$ 树的数量和高度指营巢树前 后左右各 $5 \mathrm{~m}$ 范围内的乔木的数量和平均高度;人 类干扰程度指营巢地人类出现的频率和活动强度 (分为 10 个级别, 深山无人区为 0 ,城镇市区为 9)。 同时, 我们在陕西省洋县境内随机选择 $10 \times 10 \mathrm{~m}^{2}$ 的样方 35 个, 测量同样的参数 (其中巢树高度应用 样方内乔木的平均高度, 巢向和巢上郁闭度的取值 分别为 $0 \sim 360^{\circ}$ 和 $0 \sim 100 \%$ 间均匀分布的随机值）, 作为对照样方。

然后,我们应用资源选择函数计算朱噮对每个 生境参数的选择系数,分析不同生境因子的影响程 度。同时, 我们对这 11 个生境参数进行主成分分 析，研究两种不同的多元统计方法的异同。最后，根 据我们以前整理的朱噮生境资料(李欣海等,1999) 和其资源选择函数，计算朱噮在洋县的巢址选择的 概率。

\section{2 资源选择函数}

资源选择函数描述了物种使用栖息地资源的偏 好，它可以用已利用资源与可利用资源的比率来计算。 对于生境中的一种资源 $i$ 物种对它的选择率为：

$$
w_{i}=o_{i} / \pi_{i}
$$

其中 $o_{i}$ 是资源 $i$ 中被使用的比例 , $\pi_{i}=a_{i} / a_{+}, a_{+}$是 所有可供使用的资源单位, $a_{i}$ 是其中资源 $i$ 可以被 使用的单位。

由于物种对生境的选择往往受食物、遮蔽物和水 热条件等多种因素的制约，所以资源选择函数一般表 现为一个包括多个独立生境变量的线性对数模型：

$$
w(x)=\exp \left(\beta_{0}+\beta_{1} x_{1}+\beta_{2} x_{2}+\cdots+\beta_{k} x_{k}\right)
$$

数。那么, 物种对生境的选择概率为:

$$
\tau(x)=\exp \left(\beta_{0}+\beta_{1} x_{1}+\beta_{2} x_{2}+\cdots+\beta_{k} x_{k}\right) /[1
$$

$\left.+\exp \left(\beta_{0}+\beta_{1} x_{1}+\beta_{2} x_{2}+\cdots+\beta_{k} x_{k}\right)\right]$

当 $\tau(x)$ 的取值为 0 或 1 时，即表示选择或不选择 时, 选择系数 $\beta$ 可以由逻辑斯蒂回归系数来估计。

逻辑斯蒂回归在 1967 年首次用于多变量分析 (Hosmer \& Lemeshow ,1989) ，现在它是研究二值响 应变量 (例如, 有和无) 或有序响应变量与一组自变 量之间关系的一种标准统计方法, 目前在大多数统 计软件 (如 SAS ,SPSS 等) 中都有固定的模块来实 现。我们使用 SAS 的 Logistic 过程进行这一分析 ( SAS ,1996)。

\section{2 结果}

因为应用逻辑斯蒂回归拟合资源选择函数变量 的选择系数是以变量相互独立为前提的 (Boyce \& McDonald ,1999；Lennon ,1999)，所以我们对 11 个 生境变量进行了相关分析 (表 1 )。在变量两两比较 的 50 个相关系数中 绝对值大于 0.5 的只有 4 个。

我们把所有参数都进行了标准化, 这样参数间 没有量纲的差异, 计算出来的选择系数可以相互比 较。结果朱鿷巢址的选择概率与营巢树的高度、附 近水田面积、周围树木的高度呈正相关，与坡位、人 类干扰程度、海拔高度呈负相关 (表 2 )。朱噮巢址 选择的资源选择函数为：

$\operatorname{logit}(p)=0.94-3.81 \times$ 坡位 $+2.91 \times$ 营巢 树高度 $-2.58 \times$ 干扰程度 $+2.12 \times$ 附近水田面积 $1.97 \times$ 海拔高度......

如前所述, 物种对生境的选择概率为:

$P=\mathrm{e}^{\operatorname{logit}(p)} /\left(1+\mathrm{e}^{\operatorname{logit}(p)}\right)$

根据以前整理的洋县生境数据, 我们以 $500 \times$ $500 \mathrm{~m}^{2}$ 的样方尺度计算了朱僉吕巢址选择的概率 (图 1) 结果同我们以前栖息地适宜度的研究 (李欣海 等,1999) 大体一致,但仍有部分差异。

通过对朱噮巢址生境因子的主成分分析发现， 11 个主成分分布比较平均 (表 3 ) ,其中第一主成分 中海拔高度、附近水田面积和人类干扰程度所占比 重较大，第二主成分中营巢树高度和样方内树木的 高度所占比重较大 (表 4 )。 
表 2 朱鹮巢址选择函数中生境因子的选择系数

Table 2 Selection coefficients in the nest site selection function of Crested Ibis

\begin{tabular}{|c|c|c|c|c|}
\hline $\begin{array}{c}\text { 生境因子 } \\
\text { Habitat factors }\end{array}$ & $\begin{array}{c}\text { 选择系数 } \\
\text { Selection coefficients }\end{array}$ & $\begin{array}{c}\text { 标准误 } \\
\text { Standard Error }\end{array}$ & $\begin{array}{c}\text { Wald 卡方检验值 } \\
\text { Wald Chi-square }\end{array}$ & $\begin{array}{c}\text { 概率 } \\
P\end{array}$ \\
\hline 海拔高度 Altitude (m) & -1.9658 & 1.2496 & 2.4747 & 0.1157 \\
\hline 附近水田面积 Area of paddy nearby $\left(\mathrm{hm}^{2}\right)$ & 2.1207 & 1.2657 & 2.8074 & 0.0938 \\
\hline 人类干扰程度 Human disturbance & -2.5818 & 1.1931 & 4.6822 & 0.0305 \\
\hline $\begin{array}{l}\text { 周围 } 100 \mathrm{~m}^{2} \text { 树的数量 } \\
\text { Number of trees within } 100 \mathrm{~m}^{2}\end{array}$ & -0.5207 & 0.7924 & 0.4318 & 0.5111 \\
\hline $\begin{array}{l}\text { 周围 } 100 \mathrm{~m}^{2} \text { 树的高度 } \\
\text { Average height of trees within } 100 \mathrm{~m}^{2}(\mathrm{~m})\end{array}$ & 1.5717 & 0.8923 & 3.1024 & 0.0782 \\
\hline 坡位 Nest position on the slope & -3.8135 & 1.7957 & 4.5098 & 0.0337 \\
\hline 坡向 Slope aspect (Degree) & 1.305 & 0.8914 & 2.1436 & 0.1432 \\
\hline 坡度 Slope degree & -0.3428 & 0.8458 & 0.1642 & 0.6853 \\
\hline 营巢树高度 Height of nest tree (m) & 2. 9057 & 1.2363 & 5.5239 & 0.0188 \\
\hline 巢向 Nest orientation (Degree) & -0.2738 & 0.7353 & 0.1386 & 0.7097 \\
\hline 巢上郁闭度 Coverage above the nest & 1.3454 & 1.1008 & 1.4937 & 0.2216 \\
\hline
\end{tabular}

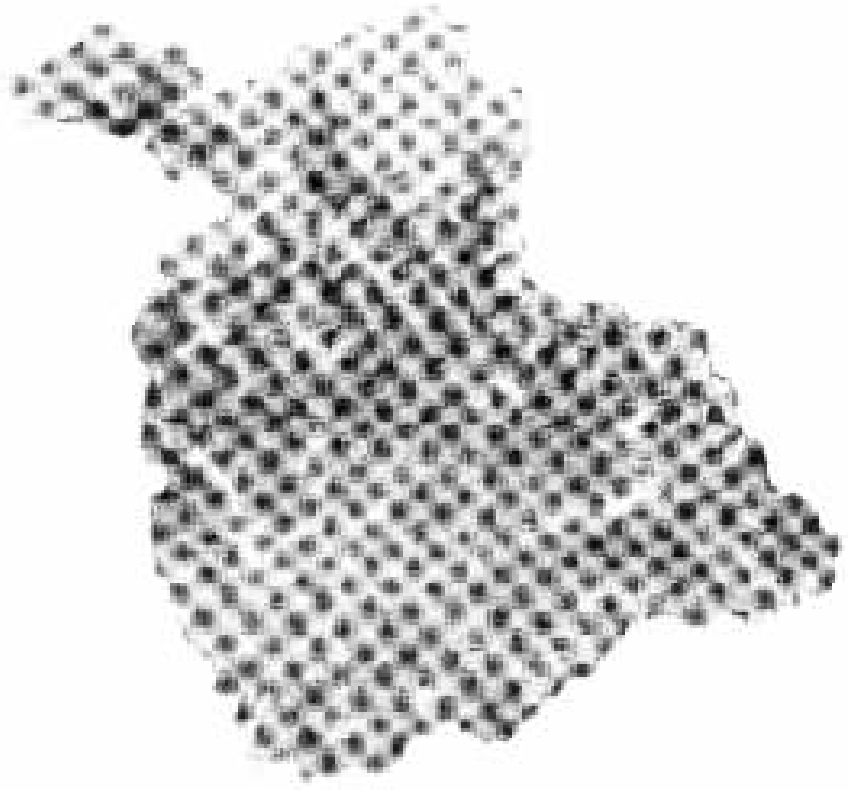

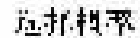

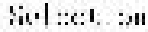 \\ pwilitis
}

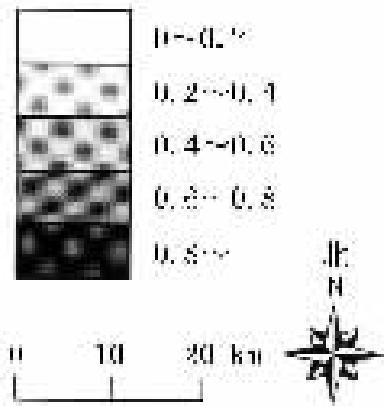

图 1 在陕西省洋县朱嗗鸟巢址选择的概率

Fig. 1 The probability of nest site selection of Crested Ibis in Yang County, Shaanxi Province

应用资源选择函数和主成分分析两种方法研究 朱噮的巢址选择，结果都显示水田面积、营巢树高 度、海拔高度和人类干扰程度对朱噮巢址选择有较 大的影响;而巢向、巢上郁闭度、坡向和坡度对朱噮 影响较小。同时, 这两种方法的结果有明显的差异。 在资源选择函数中，坡位选择系数的绝对值是最大 的，而在主成分分析中，它只对第四主成分有较大的 贡献。与坡位相反, 样方内树木的数量在主成分分 析中对第一主成分贡献较大, 而在生境选择函数中 其选择系数却很低。

\section{3 讨论}

\section{1 生境参数的选择}

我们考虑了营巢区域的环境、巢址周围的植被 和地形以及营巢树特征, 从这三个尺度中选择了 11 个因素进行分析。不同因素对朱噮的影响方式不 同。朱噮在繁殖前期向高海拔迁移,非常类似其他 候鸟的繁殖迁徙,所以朱睘对海拔高度有明显的选 择性。在繁殖期朱噮主要以水田为受食地, 足够的 水田面积是满足其生存和繁殖的关键因素。朱噮对 
表 3 影响朱噮巢址选择的生境因子的主成分分析的特征 值表

Table 3 The PCA eigenvalues for habitat factors that affect nest site selection of Crested Ibis

\begin{tabular}{cccc}
\hline $\begin{array}{c}\text { 主成分 } \\
\text { Compositions }\end{array}$ & $\begin{array}{c}\text { 特征值 } \\
\text { Eigenvalue }\end{array}$ & $\begin{array}{c}\text { 贡献率 } \\
\text { Contribution }\end{array}$ & $\begin{array}{c}\text { 累计贡献率 } \\
\text { Cumulative contribution }\end{array}$ \\
\hline 1 & 3.06418 & 0.278562 & 0.27856 \\
2 & 1.92311 & 0.174828 & 0.45339 \\
3 & 1.25661 & 0.114237 & 0.56763 \\
4 & 1.19907 & 0.109006 & 0.67663 \\
5 & 1.04933 & 0.095394 & 0.77203 \\
6 & 0.89886 & 0.081715 & 0.85374 \\
7 & 0.49890 & 0.045355 & 0.89910 \\
8 & 0.42366 & 0.038514 & 0.93761 \\
9 & 0.30046 & 0.027314 & 0.96492 \\
10 & 0.22464 & 0.020422 & 0.98535 \\
11 & 0.16119 & 0.014654 & 1.00000 \\
\hline
\end{tabular}

人类活动非常敏感,一旦发现人类在附近活动便马 上飞走。虽然朱睘能接受经常在其附近活动的农 民，而且喜欢跟在犁地的牛后面受食刚刚翻出的虫 子,但总体上人类对它的干扰还是比较大。朱鹮的 巢一般搭在树林中,偶尔也选择独立的乔木作营巢 树。朱㛱巢址的坡向和坡度主要影响附近植被的长 势, 进而影响巢的开阔度。朱愄总是在地面受食 坡 位影响朱揋返巢所飞行的高度,也影响朱噮的视野。 巢向和巢上的郁闭度影响巢表面光照和温度,而且 巢上的郁闭度可能影响其空中天敌 (如鹰、隼等) 的 威胁程度。朱鹆总是选择高大的乔木营巢, 不但为 了支撑住巢，而且如果树足够粗，便可以防止蛇等天 敌上树。

应用资源选择函数分析朱噮对不同生境因子的
偏好, 发现只有营巢树高度、坡位和人类干扰程度的 选择系数比较可靠,有统计学意义 $(p<0.05)$ (表 2)。样方内树木的平均高度、海拔和附近水田面积 的选择系数变异性稍大, 其他参数则没有规律。

\section{2 资源选择函数与主成分分析的差异}

资源选择函数与主成分分析对营巢地的坡位和 植被密度的分析结果有较大的差异。回溯原始数 据, 发现在 35 个巢址中 28 个位于山坡中部, 而对照 35 个样方中只有 11 个位于山坡中部, 即朱噮对山 坡中部的选择率 $w_{i}$ 为 2.55 , 因此朱鹆对山坡中部 有明显的选择性。资源选择函数较好地体现了这种 偏好, 而主成分分析只涉及 35 个巢址的数据, 没有 对比巢址和其他地点的差异。对于植被密度, 朱彋 在各种情况下 (样方内乔木数量 1 至 16 棵) 都曾营 巢,没有表现出明显的偏好，而在主成分分析中植被 密度却在一定程度上反映了多种生境因子的整体特 征。

主成分分析是考察多个数值变量相关性的一种 多元统计方法, 它研究如何通过少数几个主成分 (即原始变量的线性组合) 来解释多变量的方差 协方差结构。具体地说, 是导出少数几个主成分, 它 们尽可能多地保留了原始变量的信息, 且彼此间又 不相关。资源选择函数应用已利用资源和可利用资 源的比值确定物种的生境选择偏好, 应用逻辑斯蒂 回归估计选择系数。逻辑斯蒂回归研究二值响应变 量或有序响应变量与一组自变量之间关系,可以拟 合多种生境因子与物种对生境选择和不选择的关 系。因此, 这两种方法在研究野生动物的栖息地选

表 4 影响朱噮巢址选择的生境因子对主成分的贡献

Table 4 The contribution to the principal components by habitat factors that affect nest site selection of Crested Ibis

\[ \begin{array}{c}\text { 生境因子 } \\ \text { Habitat factors }\end{array} \]
海拔高度 Altitude $(\mathrm{m})$
附近水田面积 Area of paddy nearby $\left(\mathrm{hm}^{2}\right)$
人类干扰程度 Human disturbance
周围 $100 \mathrm{~m}^{2}$ 树的数量 Number of trees within $100 \mathrm{~m}^{2}$
周围 $100 \mathrm{~m}^{2}$ 树的高度 Average height of trees within $100 \mathrm{~m}^{2}(\mathrm{~m})$
坡位 Nest position on the slope
坡向 Slope aspect ( Degree)
坡度 Slope degree
营巢树高度 Height of nest tree $(\mathrm{m})$
巢向 Nest orientation (Degree)
巢上郁闭度 Coverage above the nest

\begin{tabular}{cr}
1 & \multicolumn{1}{c}{2} \\
-0.481 & -0.20 \\
0.42 & 0.31 \\
0.383 & 0.23 \\
0.387 & -0.06 \\
-0.317 & 0.46 \\
-0.089 & 0.14 \\
-0.215 & -0.06 \\
0.304 & -0.00 \\
-0.208 & 0.56 \\
-0.071 & 0.30 \\
0.023 & -0.37
\end{tabular}

主成分 Compositions

\begin{tabular}{rrrr}
3 & \multicolumn{1}{c}{4} & \multicolumn{1}{c}{5} & \multicolumn{1}{c}{6} \\
-0.089 & -0.135 & 0.171 & 0.103 \\
-0.079 & 0.152 & 0.073 & -0.089 \\
-0.199 & -0.218 & 0.199 & 0.183 \\
0.421 & 0.264 & 0.168 & -0.062 \\
-0.061 & 0.038 & -0.216 & -0.019 \\
0.001 & 0.768 & 0.138 & 0.426 \\
0.669 & -0.106 & 0.184 & 0.379 \\
0.061 & -0.417 & -0.176 & 0.619 \\
-0.162 & -0.093 & 0.066 & 0.293 \\
0.179 & -0.23 & 0.715 & -0.291 \\
-0.506 & 0.061 & 0.5 & 0.255
\end{tabular}


择中各有侧重, 其中资源选择函数在研究动物的生 境偏好方面有明显的优势。

\section{3 对照样方的选择}

应用资源选择函数，对照样方的选择非常重要。 对照样方表示物种可能选择但还没有选择的生境。 资源选择函数通过比较物种选择的生境和未选择的 生境，统计出物种对不同生境因子的偏好。一般假 定整个研究区域都可能是物种的生境，因此对照样 方应该反映整个区域的特征。为了反映整体的特 点理论上取样数越多越好。而实际上限于工作量, 一般只选取少数样方。为了保证样方的无偏性, 人 们通常随机选择样方。而当取样数不是足够大时, 随机样方的属性往往与整体的属性有相当的差距。 为了减少差距, 我们采取了按比例取样的方法。以 海拔高度为例，我们根据不同海拔高度生境面积的 比例，确定特定海拔的对照样方数（表 5)，然后根据 样方数再随机取样, 如在海拔高度 $600 \sim 800 \mathrm{~m}$ 的生 境中随机选择 8 个样方。这样选择的对照样方体现 了整个洋县的生境特征,符合朱揋在整个洋县境内 随机选择营巢地的假设。对于其他参数, 我们也作 了相同的处理。

表 5 根据洋县不同海拔高度生境面积的比例选择对照样 方

Table 5 Selecting control plots according to the proportion of area of habitats at different elevation

\begin{tabular}{rrrc}
\hline $\begin{array}{c}\text { 海拔高度 } \\
\text { Altitude }(\mathrm{m})\end{array}$ & $\begin{array}{c}\text { 面积 } \\
\text { Areas }\left(\mathrm{km}^{2}\right)\end{array}$ & $\begin{array}{c}\text { 比例 } \\
\text { Proportion }\end{array}$ & $\begin{array}{c}\text { 对照样方数量 } \\
\text { Numbers of control } \\
\text { plots }\end{array}$ \\
\hline $0 \sim 200$ & 0.550 & 0.0002 & 0 \\
$200 \sim 400$ & 1.241 & 0.0004 & 0 \\
$400 \sim 600$ & 684.610 & 0.2231 & 8 \\
$600 \sim 800$ & 735.436 & 0.2396 & 8 \\
$800 \sim 1000$ & 388.029 & 0.1264 & 4 \\
$1000 \sim 1200$ & 359.569 & 0.1172 & 4 \\
$1200 \sim 1400$ & 329.058 & 0.1072 & 3 \\
$1400 \sim 1600$ & 264.971 & 0.0863 & 2 \\
$1600 \sim 1800$ & 159.881 & 0.0521 & 1 \\
$1800 \sim 2000$ & 75.368 & 0.0246 & 1 \\
$>2000$ & 70.352 & 0.0228 & 4 \\
\hline
\end{tabular}

\section{4 参数的独立性}

我们选择的 11 个生境因子独立性较好, 但仍有 几个参数相关性大于 0.5 。其中海拔高度与水田面 积, 海拔高度与样方内乔木数量、水田面积与人类干 扰程度相关程度较大 (表 1 )。因为这些相关的参数
对朱彋的影响方式明显不同,所以我们依然保留了 这些参数。营巢树的高度与样方内乔木平均高度相 关程度也较大。我们选择这两个参数的目的之一是 研究朱鸸对营巢树高度的选择性, 结果营巢树显著 比周围的树木高 (成对双样本 $t$-检验: $t=9.98, d f=$ $34, p<0.0001$ )。我们去除了样方内乔木高度这个 参数重新计算, 结果其他生境因子的选择系数变化 不大。

\section{5 生境参数的选择系数}

资源选择函数中生境参数选择系数的大小表示 偏好程度。其中海拔高度的选择系数为负值, 这是 因为洋县的平均海拔高度为 $976 \mathrm{~m}$, 而朱鹘巢址的 平均海拔高度为 $894 \mathrm{~m}$, 所以从整个洋县来看, 朱鹮 倾向于选择较低的生境营巢。这个结果似乎与传统 的认识相矛盾。因为在繁殖期, 朱愄是从海拔较低 的汉中盆地飞到地势较高的山区的，在生境选择上 明显是倾向于更高的海拔。因为朱㟪可以轻易飞遍 洋县全境，所以我们可以从整个栖息地的角度来考 虑: 在繁殖期朱噮选择略低于平均海拔高度的生境, 而在繁殖前期朱噮选择了最低的生境。作为一种依 赖于湿地的水鸟,朱䰹的这种选择不言而喻。我们 以往仅在朱鹘的活动区内取样, 进行栖息地研究, 因 为缺乏对不适宜生境的对照研究, 结果很难反映出 朱愄对特定生境的偏好。资源选择函数需要对照样 方, 要求从任何物种可能到达地区随机取样, 正是为 了客观地计算物种对不同生境因子的偏好。

朱槶对坡位的选择也为负值 (该值的正负完全 取决于人为赋值的大小顺序), 同样是因为洋县有 大面积的平地 (坡位值为 3) , 所以朱愄营巢地的坡 位值比洋县的平均值小。

\section{6 对朱買保护的建议}

鉴于水田面积、营巢树高度、海拔高度和人类干 扰程度等生境因子对朱鲄的巢址选择非常重要, 而 繁殖期是朱噮种群恢复的关键时期, 所以在朱噮的 保护工作中，我们应该做到以下几点: 加强对水田 (尤其是冬水田) 的管理, 维持一定的面积,减少农 药和化肥的使用; 保护森林, 严禁乱砍滥伐; 减少人 为干扰。在繁殖期保护工作的重点,应当在海拔 $894 \pm 176 \mathrm{~m}$ (表 1) 的地区。对于朱鹋的再引入工 程, 其栖息地必须在水田 (或其他湿地) 的面积、树 木的高度、人类干扰程度和海拔高度等几方面充分 满足朱噮的需求。 


\section{参考文献}

高中信, 陈华豪, 陈化鹏, 罗理扬, 刘景全, 金昆, 1992. 动物生 态学实验与实习方法. 哈尔滨: 东北林业大学出版社, 1 $\sim 280$

李欣海, 李典谟, 路宝忠, 翟天庆, 1996. 朱噮( Nipponia nippon) 种群生存力分析. 生物多样性, 1996, 4(2) : 69 77

李欣海, 李典谟, 丁长青, 曹永汉, 卢西荣, 傅文凯, 马志军, 路 宝忠, 翟天庆, 1999. 朱噮 (Nipponia nippon) 栖息地质量 的初步评价. 生物多样性, 1999, 7(3): 161 169

马志军, 李文军, 王子健, 2000. 丹顶鹤的自然保护. 北京: 清 华大学出版社

Alldredge J R and Ratti J T, 1986. Comparison of some statistical techniques for analysis of resource selection. Journal of Wildlife Management, 50: $157 \sim 165$

Alldredge J R and Ratti J T, 1992. Further comparison of some statistical techniques for analysis of resource selection. Journal of Wildlife Management, 56: $1 \sim 9$

Boyce M S and McDonald L L, 1999. Relating populations to habitats using resource selection functions. Trends in Ecology and Evolution, 14:268 272

Byers C R, Steinhorst R K and Krausman P R, 1984. Clarification of a technique for analysis of utilization-availability data. Journal of Wildlife Management, 48: $1050 \sim 1053$

Hosmer Jr D W and Lemeshow S, 1989. Applied Logistic Regression. New York: John Wiley \& Sons

Lennon J J, 1999. Resource selection functions: taking space seriously? Trends in Ecology and Evolution, 14: $399 \sim 400$

Liang Q and Thomson A J, 1994. Habitat-abundance relationships of the earthworm Eisenia rosea (Savigny) (Lumbricidae), using principal component regression analysis. $\mathrm{Ca}$ nadian Journal of Zoology, 72(7): 1354 1361

Manly B F J, McDonald L L and Thomas D L, 1993. Resource Selection by Animals: Statistical Design and Analysis for Field Studies. London: Chapman \& Hall

Mladenoff D J and Sickley T A, 1998. Assessing potential gray wolf restoration in the United States: a spatial prediction of favorable habitat and potential population levels. Journal of Wildlife Management, 62: $1 \sim 10$

Neu C W, Byers C R and Peek J M, 1974. A technique for analysis of utilization-availability data. Journal of Wildlife Management, 38: $541 \sim 545$

Pogue D W and Schnell G D, 1994. Habitat characterization of secondary cavity-nesting birds in Oklahoma. Wilson Bulletin, 106(2): $203 \sim 226$

SAS Institute, 1996. SAS for Windows. Release 6. 12. Cary, North Carolina. USA: SAS Institute

Wenny D G, Clawson R L, Faaborg J and Sheriff S L, 1993. Population density, habitat selection and minimum area requirements of three forest-interior warblers in central Missouri. Condor, 95(4): $968 \sim 979$

(责任审稿人：丁 平;责任编辑 : 问文杰) 